\title{
Hadronic freeze-out following a first order hadronization phase transition in ultrarelativistic heavy-ion collisions
}

\author{
S.A. Bass \\ Department of Physics \\ Duke University \\ Durham, NC, 27708-0305, USA \\ A. Dumitru \\ Department of Physics \\ Yale University \\ New Haven, CT, 06520, USA \\ Institut für Theoretische Physik \\ Johann Wolfgang Goethe Universität \\ Robert Mayer Str. 8-10 \\ D-60054 Frankfurt am Main, Germany
}

M. Bleicher, L. Bravina, E. Zabrodin, H. Stöcker and W. Greiner

\begin{abstract}
We analyze the hadronic freeze-out in ultra-relativistic heavy ion collisions at RHIC in a transport approach which combines hydrodynamics for the early, dense, deconfined stage of the reaction with a microscopic non-equilibrium model for the later hadronic stage at which the hydrodynamic equilibrium assumptions are not valid. With this ansatz we are able to self-consistently calculate the freeze-out of the system and determine space-time hypersurfaces for individual hadron species. The space-time domains of the freeze-out for several hadron species are found to be actually four-dimensional, and differ drastically for the individual hadrons species. Freeze-out radii distributions are similar in width for most hadron species, even though the $\Omega^{-}$is found to be emitted rather close to the phase boundary and shows the smallest freezeout radii and times among all baryon species. The total lifetime of the system does not change by more than $10 \%$ when going from SPS to RHIC energies.
\end{abstract}


Ultra-relativistic heavy ion collisions are the only means available to investigate highly excited dense nuclear matter under controlled laboratory conditions. In such collisions it is sought to recreate a Quark Gluon Plasma (QGP), the highly excited state of primordial matter which is believed to have existed shortly after the creation of the universe in the Big Bang (for recent reviews on the QGP, we refer to [四).

Transport theory has been among the most successful approaches applied to the theoretical investigation of relativistic heavy ion collisions. Microscopic transport models attempt to describe the evolution of the heavy ion reaction from some initial state up to the freeze-out of the newly produced particles on the basis of elementary interactions. The basic constituents in such models are either hadrons [2,3] or partons 团]. At RHIC energies, however, both, partonic and hadronic, degrees of freedom might be equally important and have both to be treated explicitly [5]. However, in such microscopic transport models, the QG-matter to hadron matter transition, i.e. the hadronization stage, has to be modeled in an ad-hoc fashion, whereas hydrodynamic approaches [6 10] incorporate this as a phase transition. This can be done in a consistent way, respecting the laws of thermodynamics (which is not always the case in microscopic transport models). The drawback of hydrodynamics, however, is that in the later reaction stages the basic hydrodynamical assumptions break down. For the freeze-out of the system a decoupling (freeze-out) hyper-surface must be specified (or fine-tuned to existing data).

In this letter, we use boost-invariant hydrodynamics to model a first order phase transition from a QGP to a hadronic fluid, and combine it with a non-equilibrium microscopic transport calculation for the later, purely hadronic stages of the reaction. With this ansatz we are able to self-consistently calculate the freeze-out of the system: no decoupling hypersurface is imposed by hand, but the space-time points are rather determined by an interplay between the (local) expansion scalar $\partial u$ [1],10] (where $u$ is the collective flow four-velocity), the relevant elementary cross sections, and the equation of state (EoS), which actually changes dynamically as more and more hadron species decouple.

Let us first briefly describe the hydrodynamical model employed here: For a more detailed discussion we refer to refs. [9, 12,13]. For simplicity, boost-invariant longitudinal flow [6] is assumed. For ultrarelativistic collisions, this should be a reasonable first approximation in the central rapidity region. Cylindrically symmetric transverse expansion is superimposed. For $T>T_{C}=160 \mathrm{MeV}$ the well-known MIT bag model equation of state [14 is used, assuming for simplicity an ideal gas of quarks, anti-quarks (with masses $m_{u}=m_{d}=0$, $m_{s}=150 \mathrm{MeV}$ ), and gluons. For $T<T_{C}$ an ideal hadron gas is employed that includes the complete hadronic spectrum up to a mass of $2 \mathrm{GeV}$. At $T=T_{C},\left(\mu_{B}=\mu_{S}=0\right)$ we require that both pressures are equal, which fixes the bag constant to $B=380 \mathrm{MeV} / \mathrm{fm}^{3}$. By construction the EoS exhibits a first order phase transition (which is also expected in QCD for the quark-hadron phase transition in the case of three quark flavors). 
The model reproduces the measured $p_{T^{-}}$and $m_{T^{-}}$spectra of hadrons at the SPS, when assuming that hydrodynamic flow sets in on the proper time hyperbola $\tau_{i}=1 \mathrm{fm} / \mathrm{c}$ [9], 12]. This is a value conventionally assumed in the literature, cf. e.g. [6]. Due to the higher parton density at midrapidity, thermalization may be reached earlier at RHIC [15]. As in refs. [9, 12], we assume here $\tau_{i}=R_{T} / 10=0.6 \mathrm{fm}$. The effects of variations of $\tau_{i}$ and $T_{C}$ will be discussed in a future publication [13]. Moreover, we use the initial average energy and baryon densities $\bar{\epsilon}\left(\tau_{i}\right)=20 \mathrm{GeV} / \mathrm{fm}^{3}$ and $\bar{\rho}\left(\tau_{i}\right)=2.3 \rho_{0}$, which lead to $d N_{B} / d y=25$ and $\bar{s} / \bar{\rho}_{B}=205$ (a bar symbols an average over the transverse plane). The initial energy and net baryon densities are assumed to be distributed in the transverse plane according to a so-called "wounded nucleon" distribution $\propto \frac{3}{2} \sqrt{1-r_{T}^{2} / R_{T}^{2}}$, with transverse radius $R_{T}=6 \mathrm{fm}$. For this set of parameters, the initial transverse energy at midrapidity is $\mathrm{d} E_{T} / \mathrm{d} y=1.3 \mathrm{TeV}$. Due to the work performed by the isentropic expansion, it decreases to $716 \mathrm{GeV}$ on the hadronization hypersurface. The microscopic treatment of the hadronic dynamics following hadronization (see below) yields $\mathrm{d} E_{T} / \mathrm{d} y=714 \mathrm{GeV}$ at kinetic freeze-out. Thus, the late hadronic evolution at RHIC energy is not isentropic.

After specifying the initial conditions and the EoS, we determine numerically the hydrodynamical solution between the $\tau=\tau_{i}$ hyperbola and the hadronization hypersurface, where we apply the Cooper-Frye formula [16] to obtain the hadron spectra. However, in contrast to the usual procedure we do not integrate over the hypersurface, because we further on also need the space-time distribution of hadrons emerging from the mixed phase, not only their momentum-space distributions [12]. The ensemble of hadrons thus generated is then used as initial condition for the non-equilibrium microscopic transport model Ultra-relativistic Quantum Molecular Dynamics (UrQMD) [3, 17]. The UrQMD model contains hadronic (and string) degrees of freedom - all hadronic states can be produced in string decays, schannel collisions or resonance decays. Tabulated and parameterized experimental cross sections are used when available. Resonance absorption, decays and scattering are handled via the principle of detailed balance. The UrQMD model has been extensively tested in the SIS, AGS and SPS energy domain and provides a robust description of hadronic heavy-ion physics phenomenology. An extensive description of the model, as well as comparisons with various available data can be found in [3, 17].

During the mixed phase the system is either described locally within the hydrodynamical framework (as long as a non-zero fraction of the fluid in the cell consists of quark and gluons) or within the microscopic transport (in the case of pure hadronic matter). Therefore there exists a time interval during the reaction in which both models are applied in parallel, even though they never refer locally to the same space-time volume.

Let us now turn to the reaction dynamics of central (impact parameter $b=0 \mathrm{fm}$ ) $\mathrm{Au}+\mathrm{Au}$ collisions at RHIC energies $(\sqrt{s}=200 \mathrm{GeV}$ per incident colliding nucleon-pair). We start with the analysis of the freeze-out hypersurfaces of pions and nucleons, the most abundant 
meson and baryon species in the system, restricting ourselves to the central rapidity region $y=\left|y_{C M}\right| \leq 0.5$. Figure [ 1 shows the freeze-out time distributions and the transverse radius distributions for both, pions and nucleons. The top row shows the result of a pure hydrodynamical calculation up to complete hadronization, with subsequent hadronic decays, but without hadronic reinteraction. The bottom row shows the same calculation with full microscopic hadronic dynamics added.

The freeze-out characteristics of both, pions and especially nucleons, are significantly modified due to the hadronic interaction phase. The average transverse freeze-out radius of the pions changes from 7.8 to $9.5 \mathrm{fm}$ and that of the protons doubles from 5.4 to 11.3 $\mathrm{fm}$. Their respective average freeze-out times change from 17.2 to $23.1 \mathrm{fm} / \mathrm{c}$ (pions) and from 11.3 to $25.8 \mathrm{fm} / \mathrm{c}$ (protons). As the meson multiplicity in the system is fifty times larger than the baryon multiplicity, baryons propagate through the relativistic meson gas - they may act as probes of this highly excited meson medium. Thus, a first estimate of the duration of the hadronic phase is $\Delta \tau \approx 13 \mathrm{fm} / \mathrm{c}$. Its transverse spatial extent is on the order of $\Delta r_{T} \approx 6 \mathrm{fm}$.

The Hydro+UrQMD model predicts a space-time freeze-out picture which is drastically different from that usually employed in the hydrodynamical model, e.g. in refs. [8 [1, 19, 20]: Freeze-out here is found to occur in a four-dimensional region within the forward lightcone [21] rather than on a three-dimensional "hypersurface" in space-time [16]. Similar results have also been obtained within other microscopic transport models [18] when the initial state was not a quark-gluon plasma. This finding seems to be a generic feature of such models: the elementary binary hadron-hadron interactions smear out the sharp signals to be expected from simple hydro. This predicted additional fourth dimension of the freezeout domain could affect the HBT parameters considerably.

This does not mean that the momentum-distributions alone can not be calculated assuming freeze-out on some effective three-dimensional hypersurface. (For example, if interactions on the outer side of that hypersurface are very "soft", the single-particle momentum distributions will not change anymore, while the two-particle correlator does change. Thus, the freeze-out condition, e.g. the temperature, as measured by single-particle spectra and two-particle correlations [22] needs not be the same.)

The shapes of the freeze-out hypersurfaces (FOHS) show broad radial maxima for intermediate freeze-out times. Thus, transverse expansion has not developed scaling-flow (in that case the FOHS would be hyperbolas in the $\tau-r_{T}$ plane). Moreover, the hypersurfaces of pions and nucleons, and their shapes, are distinct from each other (as also found in [3.10,18,23] at the lower BNL-AGS and CERN-SPS energies). Thus, our calculation contradicts the ansatz of a unique freeze-out hypersurface for all hadrons, cf. also refs. [12, 18].

Figure 2 shows the transverse freeze-out radius distributions for $\pi, K, p, \Lambda, \Xi$ and $\Omega^{-}$. They are rather broad and similar to each other, even though the $\Omega^{-}$shows a somewhat 
narrower freeze-out distribution. The average transverse freeze-out radii are $9.5 \mathrm{fm}$ for pions, $10.2 \mathrm{fm}$ for kaons, $11.3 \mathrm{fm}$ for protons, $11.6 \mathrm{fm}$ for Lambda- and Sigma-Hyperons, $14.2 \mathrm{fm}$ for Cascades, but only $7.3 \mathrm{fm}$ for the $\Omega^{-}$. The freeze-out of the $\Omega^{-}$occurs rather close to the phase-boundary [12], due to its very small hadronic interaction cross section. This behavior could be responsible for the experimentally observed hadron-mass dependence of the inverse slopes of the $m_{T^{-}}$spectra at SPS energies [24]. For the $\Omega^{-}$, the inverse slope remains practically unaffected by the purely hadronic stage of the reaction, due to its small interaction cross section, while the flow of $p$ 's and $\Lambda$ 's increases [12]. By comparing the transverse freeze-out radii of the hydrodynamical calculation (up to hadronization, including subsequent hadronic decays, but no hadronic reinteractions) with the Hydro+UrQMD calculation, which include microscopic hadronic dynamics, the thickness $\Delta r_{\text {had }}$ of the hadronic phase can be estimated by computing the difference: $\Delta r_{h a d}=\left\langle r_{t, f r}^{\text {Hydro }+\mathrm{UrQMD}}\right\rangle-\left\langle r_{t, f r}^{\text {Hydro+had.decays }}\right\rangle$. These values for $\Delta r_{\text {had }}$ are: $1.7 \mathrm{fm}$ for pions, $3.1 \mathrm{fm}$ for kaons, $5.8 \mathrm{fm}$ for protons, lambda- and sigma-hyperons as well as cascades and $2.6 \mathrm{fm}$ for the $\Omega^{-}$.

Another issue of interest is the predicted significant increase of the lifetime of the system from SPS to RHIC energies [8]. Figure 3 shows that in our model, which exhibits a first order phase transition, there is between SPS and RHIC no difference in the freeze-out time distributions of $\pi, p$, and $\Omega^{-}$! Origin of this prediction is that we include many more states in the hadronic EoS, which speeds up hadronization considerably [9,20. Furthermore, decays of resonances (which were not treated in [8]) mask the remaining small increase of the hadronization time. Note that the multistrange $\Omega^{-}$baryons freeze out far earlier than all other baryons, as discussed already previously in the context of figure 2. The duration of the hadronic reinteraction phase, $\Delta \tau_{\text {had }}=\left\langle\tau_{f r}^{\text {Hydro }+\mathrm{UrQMD}}\right\rangle-\left\langle\tau_{f r}^{\text {Hydro+had.decays }}\right\rangle$ remains nearly unchanged, e.g. at $5.9 \mathrm{fm} / \mathrm{c}$ for pions, $8.0 \mathrm{fm} / \mathrm{c}$ for kaons, $14.5 \mathrm{fm} / \mathrm{c}$ for protons, $15.4 \mathrm{fm} / \mathrm{c}$ for hyperons and $8.0 \mathrm{fm} / \mathrm{c}$ for the $\Omega^{-}$.

Note that the lifetime of the prehadronic stage in this approach is a factor of $2-3$ longer than when employing the parton cascade model (PCM) 四泡 for the initial reaction stage. It will be interesting to check whether this is related to the first-order phase transition built into the EoS which is used here. The final transverse freeze-out radii and times (after hadronic rescattering), however, are very similar in both approaches [5].

So far, we have only discussed the kinetic freeze-out of individual hadron species, which is the most precisely determinable freeze-out quantity of the system. However, apart from the kinetic freeze-out, the chemical freeze-out of the system, which fixes the chemical composition is of interest. The top frame of figure 4 shows the time evolution of on-shell hadron multiplicities. The dark grey shaded area indicates the duration of the QGP phase whereas the light grey shaded area depicts the mixed phase (both averaged over $r_{T}$; only hadrons that have already "escaped" from the mixed phase are shown). Hadronic resonances are formed and are populated for a long time $(\approx 20 \mathrm{fm} / \mathrm{c})$. When the mixed phase ceases to 
exist, the hadron yields have not yet saturated (even if resonance decays are taken into account). This is due to inelastic hadron-hadron collisions. In particular the yield of antiprotons drops strongly - more than $60 \%$ of the baryon-anti-baryon annihilations occur after the phase-coexistence is over (c.f. the lower frame of this figure). The yields of all stable hadrons saturate at approximately $25 \mathrm{fm} / \mathrm{c}$. Only then may the system be viewed as chemically frozen-out. Since resonance decays have not been included into our estimate of the saturation time, this number may be viewed as an upper estimate of the chemical freeze-out time.

By comparing different final hadron yields resulting from the hydrodynamical calculation (up to hadronization, including subsequent hadronic decays, but no hadronic reinteractions) to that of the Hydro+UrQMD calculation, which includes microscopic hadronic dynamics, we can quantify the changes of the hadrochemical content due to hadronic rescattering: especially the multiplicities of (anti)baryons vary at least by $10 \%$, those of protons and antiprotons even up to $30 \%(\pi:+9.3 \%, K:-5 \%, Y:+12 \%, p:-21 \%, \bar{p}:-31 \%, \bar{Y}:$ $+11 \%$ ). These changes clearly indicate that in our model chemical freeze-out of (anti)baryons and (anti-)hyperons does not occur directly at the phase-boundary. Also, note that (unlike in ideal chemical equilibrium) baryon number is "shuffled" from non-strange to strange baryons.

The bottom frame of figure 4 shows the rates for hadron-hadron collisions. Mesonmeson (MM) and - to a lesser extent - meson-baryon (MB) interactions dominate the dynamics in the hadronic phase. However, the $B \bar{B}$ collisions outnumber $B B$ reactions, in clear contrast to SPS. This is a consequence of the fact that the $B \bar{B}$ annihilation cross sections at small relative momenta increase faster then the total $B B$ cross section [i] ]. In the case of (approximate) baryon-antibaryon symmetry, one therefore expects more $B \bar{B}$ than $B B$ interactions, as seen in figure 1 .

All collision rates reach their maxima at the end of the mixed phase - then they decrease roughly according to a power-law. After $\approx 35 \mathrm{fm} / \mathrm{c}$, less than one hadron-hadron collision occurs per unit of time and rapidity - at this stage the system can be considered as kinetically frozen-out.

In summary, we have analyzed the hadronic freeze-out in ultra-relativistic heavy ion collisions at RHIC in a transport approach which combines hydrodynamics for the early, dense, deconfined stage of the reaction with a microscopic non-equilibrium model for the later hadronic stage at which the hydrodynamic equilibrium assumptions are not valid anymore. Within this approach we have self-consistently calculated the freeze-out of the hadronic system and accounted for the collective flow on the hadronization hypersurface generated by the QGP expansion.

We find that the space-time domains of the freeze-out for the investigated hadron species are actually four-dimensional, and differ drastically between the individual hadrons species. 
The thickness of the hadronic phase is found to be between $2 \mathrm{fm}$ and $6 \mathrm{fm}$, depending on the respective hadron species. Its lifetime is between $5 \mathrm{fm} / \mathrm{c}$ and $13 \mathrm{fm} / \mathrm{c}$, respectively. Freeze-out radii distributions are similar in width for most hadron species, even though the $\Omega^{-}$is found to be emitted rather close to the phase boundary and shows the smallest freeze-out radii and times among all baryon species. The total lifetime of the system does not change by more than $10 \%$ when going from SPS to RHIC energies. Finally, we have found in our model that chemical freeze-out of (anti-)baryons does not occur at the phase boundary and precedes the kinetic freeze-out of the system.

\section{ACKNOWLEDGMENTS}

S.A.B. is supported in part by the Alexander von Humboldt Foundation through a Feodor Lynen Fellowship, and in part by DOE grant DE-FG02-96ER40945. A.D. gratefully acknowledges a postdoctoral fellowship by the German Academic Exchange Service (DAAD) and L. Bravina is supported by the Alexander von Humboldt Foundation as a Humboldt Fellow. S.A.B. acknowledges many helpful and inspiring discussions with Berndt Müller. A.D. and S.A.B also wish to thank Ulrich Heinz for useful comments. 


\section{REFERENCES}

[1] J. Harris and B. Müller, Ann. Rev. Nucl. Part. Sci. 46 (1996) 71.

S.A. Bass, M. Gyulassy, H. Stöcker and W. Greiner, J. Phys. G25 (1999) R1, e-print hep-ph/9810281.

[2] G. Peilert, A. Rosenhauer, H. Stöcker, W. Greiner, and J. Aichelin, Mod. Phys. Lett. A3 (1988) 459.

H. Sorge, H. Stöcker, and W. Greiner, Annals of Physics 192 (1989) 266.

K. Weber, B. Blattel, V. Koch, A. Lang, W. Cassing and U. Mosel, Nucl. Phys. A515 (1990) 747.

J. Aichelin, Phys. Rep. 202 (1991) 233.

Y. Pang, T. J. Schlagel and S. H. Kahana, Phys. Rev. Lett. 68 (1992) 2743.

B. A. Li and C. M. Ko, Phys. Rev. C52 (1995) 2037.

[3] S. A. Bass, M. Belkacem, M. Bleicher, M. Brandstetter, L. Bravina, C. Ernst, L. Gerland, M. Hofmann, S. Hofmann, J. Konopka, G. Mao, L. Neise, S. Soff, C. Spieles, H. Weber, L. A. Winckelmann, H. Stöcker, W. Greiner, C. Hartnack, J. Aichelin and N. Amelin. Progr. Part. Nucl. Physics Vol. 41 (1998) 225, nucl-th/9803035.

The UrQMD source code is available via the URL:

http://www.th.physik.uni-frankfurt.de/ urqmd

[4] K. Geiger and B. Müller, Nucl. Phys. B369 (1992) 600.

K. Geiger, Phys. Rep. 258 (1995) 237.

K. Geiger, Comp. Phys. Com. 104 (1997) 70.

[5] S.A. Bass, M. Hofmann, M. Bleicher, L. Bravina, E. Zabrodin, H. Stöcker and W. Greiner, e-print nucl-th/9902055 and submitted.

[6] J.D. Bjorken, Phys. Rev. D27 (1983) 140.

K. Kajantie and L. McLerran, Nucl. Phys. B214 (1983) 261.

[7] R. B. Clare and D. Strottman, Phys. Reports 141 (1986) 179.

N. S. Amelin, L. P. Csernai, E. F. Staubo and D. Strottman, Nucl. Phys. A544 (1992) 463c.

L.P. Csernai, J.I. Kapusta, G. Kluge and E.E. Zabrodin, Z. Phys. C58 (1993) 453.

[8] D.H. Rischke and M. Gyulassy, Nucl. Phys. A597 (1996) 701, and A608 (1996) 479.

S. Bernard, D.H. Rischke, J.A. Maruhn, W. Greiner, Nucl. Phys. A625 (1997) 473.

[9] A. Dumitru and D.H. Rischke, Phys. Rev. C59 (1999) 354.

[10] C.M. Hung and E. Shuryak, Phys. Rev. C57 (1998) 1891. 
[11] I. Mishustin and L. Satarov, Sov. J. Nucl. Phys. 37 (1983) 532.

[12] A. Dumitru, S.A. Bass, M. Bleicher, H. Stöcker and W. Greiner, nucl-th/9901046

[13] S.A. Bass et al., manuscript in preparation

[14] A. Chodos, R.L. Jaffe, K. Johnson, C.B. Thorn and V.F. Weisskopf, Phys. Rev. D9 (1974) 3471.

[15] K. Geiger, Phys. Rev. D46 (1992) 4986.

[16] F. Cooper and G. Frye, Phys. Rev. D10 (1974) 186.

[17] M. Bleicher, E Zabrodin, C. Spieles, S. A. Bass, C. Ernst, S. Soff, L. Bravina, H. Weber, H. Stöcker and W. Greiner. to be published in J. Phys. G

[18] L.V. Bravina, I.N. Mishustin, N.S. Amelin, J.P. Bondorf, L.P. Csernai, Phys. Lett. B354 (1995) 196.

H. Sorge, Phys. Lett. B373 (1996) 16.

[19] Y. Sinyukov, Z. Phys. C43 (1989) 401.

S. Bernard, J.A. Maruhn, W. Greiner, and D.H. Rischke, Nucl. Phys. A605 (1996) 566. K.A. Bugaev, Nucl. Phys. A606 (1996) 559.

L.P. Csernai, Z.I. Lazar, and D. Molnar, Heavy Ion Physics 5 (1997) 467.

[20] J. Sollfrank et al., Phys. Rev. C55 (1997) 392.

J. Cleymans, K. Redlich, and D.K. Srivastava, ibid. C55 (1997) 1431.

[21] F. Grassi, Y. Hama, and T. Kodama, Z. Phys. C73 (1996) 153

[22] S. Chapman, J.R. Nix, and U. Heinz, Phys. Rev. C52 (1995) 2694.

U.A. Wiedemann, B. Tomasik, and U. Heinz, Nucl. Phys. A638 (1997) 475c.

J.R. Nix, D. Strottman, H.W. van Hecke, B.R. Schlei, J.P. Sullivan, and M.J. Murray, nucl-th/9801045.

H. Appelshäuser et al. (NA49 Collaboration), Eur. Phys. J. C2 (1998) 661.

[23] S. Pratt and J. Murray, Phys. Rev. C57 (1998) 1907

[24] H. van Hecke, H. Sorge and N. Xu, Phys. Rev. Lett. 81 (1998) 5764.

M. Bleicher, C. Spieles, C. Ernst, L. Gerland, S. Soff, H. Stöcker, W. Greiner and S.A. Bass, Phys. Lett. B447 (1999) 227. 


\section{FIGURES}

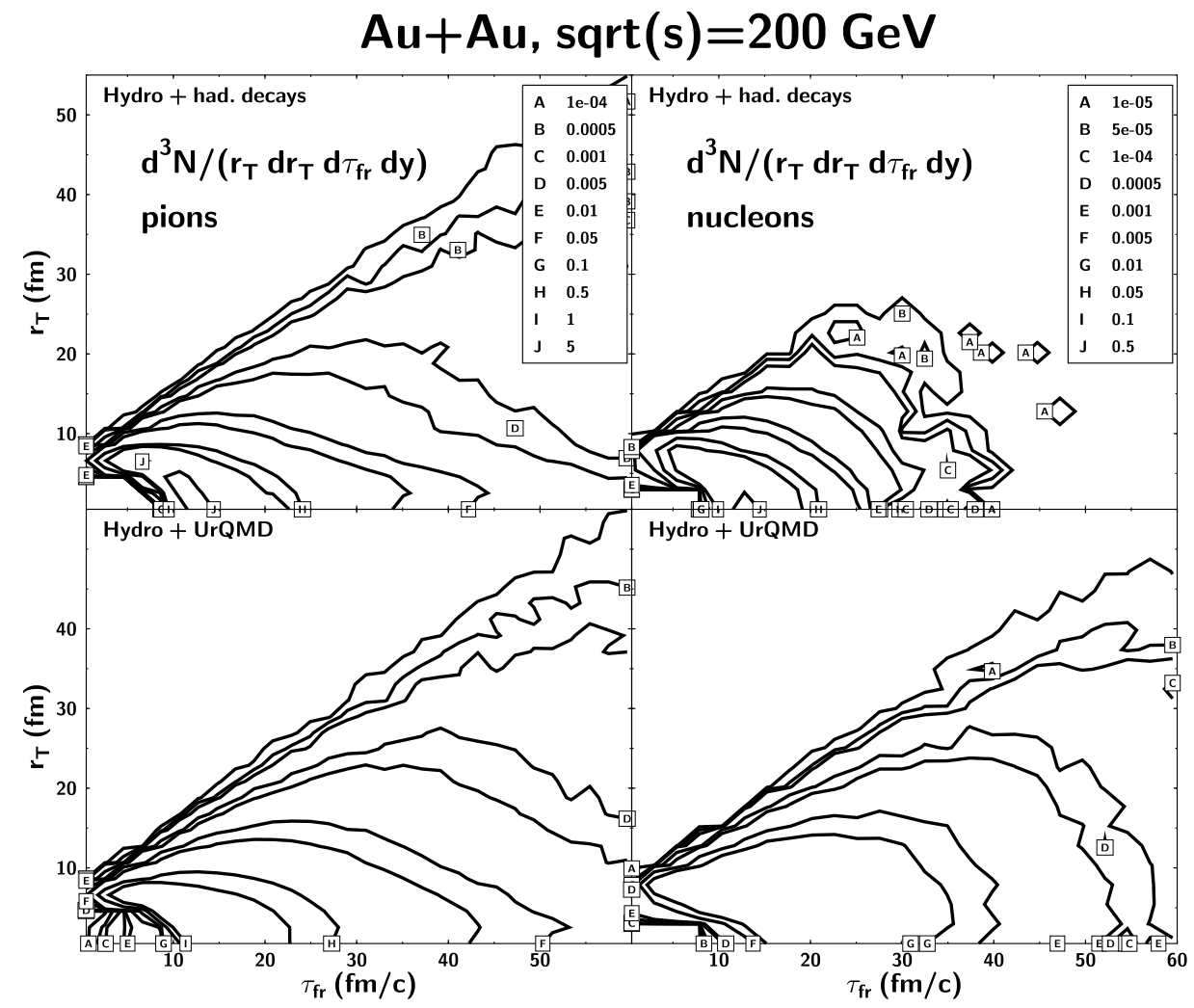

FIG. 1. Freeze-out time and transverse radius distribution $\mathrm{d}^{3} N /\left(r_{T} \mathrm{~d} r_{T} \mathrm{~d} \tau_{f r} \mathrm{~d} y\right)$ for pions (left column) and protons (right column). The top row shows the result for the pure hydro case up to hadronization with subsequent hadron resonance decays (but without hadronic reinteraction). The bottom row shows the analogous calculation, but with full microscopic hadronic collision dynamics after the hadronization. The contour lines have identical binning within each column but differ between the two columns. 


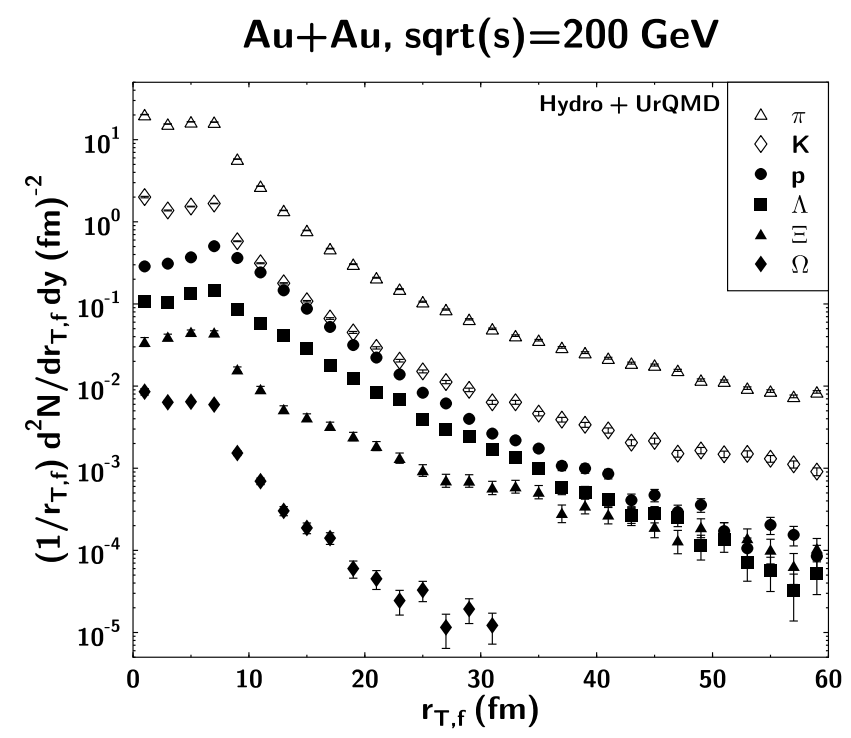

FIG. 2. Transverse freeze-out radius distributions $\mathrm{d}^{2} N / r_{T, f} \mathrm{~d} r_{T, f} \mathrm{~d} y$ for various hadron species. The distributions for $\pi, K, p, \Lambda$ and $\Xi$ are broad and similar to each other, whereas the $\Omega^{-}$exhibits a narrower freeze-out distribution.

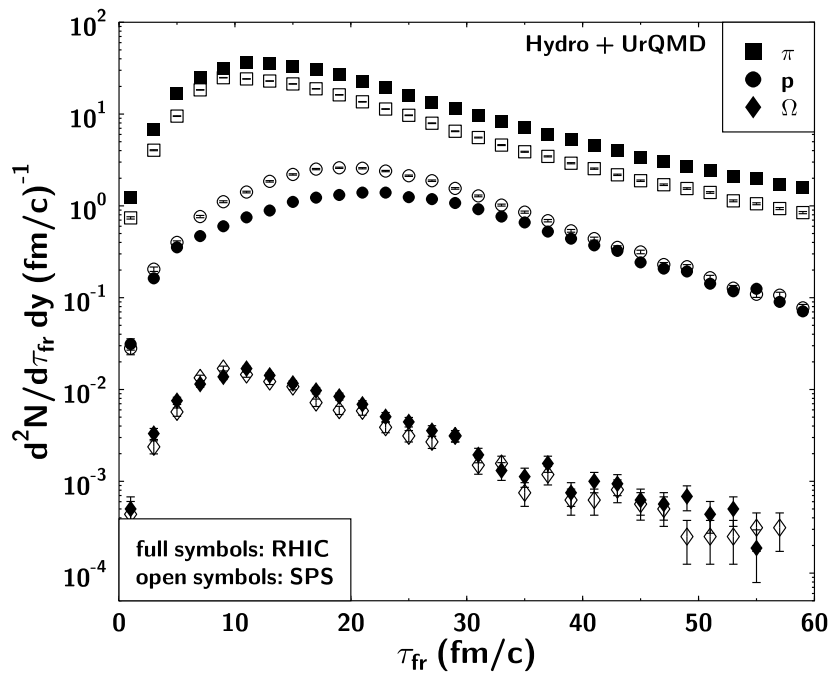

FIG. 3. Freeze-out time distributions $\mathrm{d}^{2} N / \mathrm{d} \tau_{f r} \mathrm{~d} y$ of $\pi, p$ and $\Omega^{-}$for SPS and RHIC. Apart from the different integral values there is no significant difference between the RHIC and SPS distributions, i.e. the total lifetime of the reaction is comparable. 


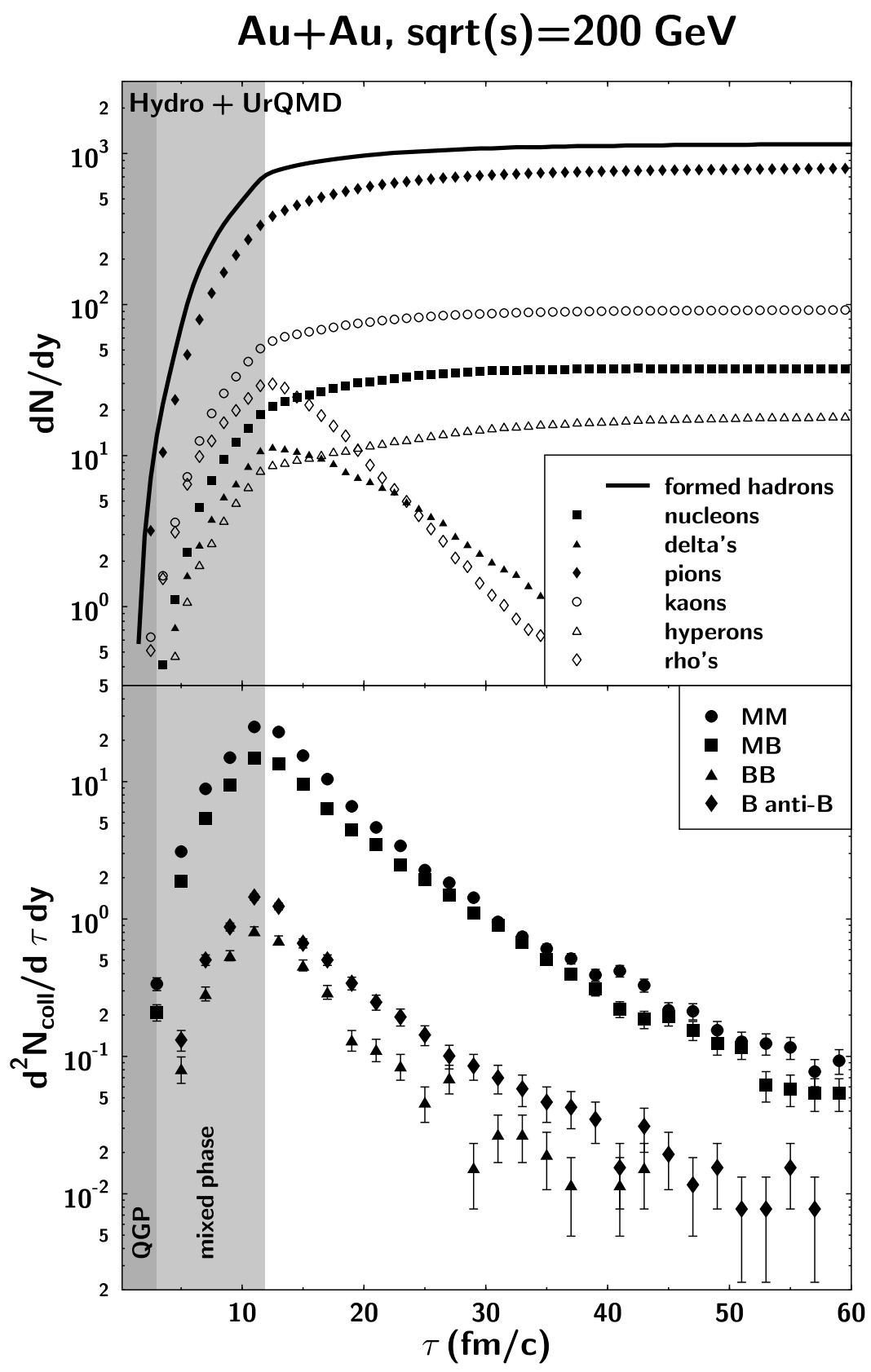

FIG. 4. Top: time evolution of on-shell hadron multiplicities (integrated over $r_{T}$ ). The dark grey shaded area shows the duration of the QGP phase whereas the light grey shaded area depicts the coexistance phase. Bottom: Hadron-hadron collision rates. 